\title{
Gestión de la innovación en las gerencias de prestación de servicios de la industria petrolera
}

\author{
Management of innovation in the management of service providing of the oil industry
}

Javier Rondón

rondonjac@gmail.com

Código ORCID: 0000-0002-7902-2215

Petróleos de Venezuela SA, Venezuela
4 Articulo recibido en julio 2019

$<$ Arbitrado en agosto 2019

< Publicado en enero 2020

\section{Resumen}

El estudio tuvo como propósito analizar la gestión de la innovación en las gerencias de prestación de servicios de la industria petrolera región Occidente. Metodológicamente se consideró de tipo descriptiva, bajo un diseño no experimental, transeccional, de campo. La población la conformo las cuatro (04) gerencias de prestación de servicios de la industria petrolera región occidente. Como técnica se empleó la encuesta mediante la aplicación de un cuestionario de 31 ítems con escala de frecuencia. La validación se realizó a través del juicio de cinco (5) expertos, y su confiabilidad a través del coeficiente Alpha de Cronbach, arrojando alta confiabilidad 0,79. Los resultados evidencian una regular gestión de la innovación, ya que existen debilidades en cuanto a las clases de innovaciones, de la misma manera una leve fortaleza en las etapas del sistema de la gestión, asimismo se detectó debilidad en los factores determinante en gestión de la innovación.

Palabras clave: Gestión de la innovación, etapas, factores e industria petrolera

\begin{abstract}
The purpose of the study was to analyze the management of innovation in the management of service provision of the oil industry in the western region. Methodologically it was considered descriptive, under a non-experimental, transectional, field design. The population was formed by the four (04) management departments for the provision of services in the oil industry in the western region. As a technique, the survey was used by applying a questionnaire of 31 items with a frequency scale. Validation was performed through the judgment of five (5) experts, and its reliability through Cronbach's Alpha coefficient, yielding high reliability of 0.79 . The results show a regular management of innovation, since there are weaknesses in terms of the types of innovations, in the same way a slight strength in the stages of the management system, also weakness was detected in the determining factors in management of the innovation.
\end{abstract}

Keywords: Innovation management, stages, factors and oil industry 


\section{INTRODUCCIÓN}

La creatividad y la innovación son elementos diferenciadores que permiten que una empresa desarrolle y mantenga su posición de liderazgo en el mercado. Los resultados de su aplicación son nuevas propuestas de servicios y productos que anticipan las expectativas de clientes exigentes que aseguran su fidelidad.

En este contexto, las organizaciones para mantenerse competitivas deben establecer estrategias que permitan un mejoramiento continuo de sus procesos, por ello, sus acciones deben estar enmarcadas en la búsqueda de la eficacia gerencial, donde puedan proporcionar productos y senvicios ajustados a las necesidades de su entorno, logrando ser cada día más competitivos. En la actualidad el mundo se encuentra sumergido en la era de la innovación, es unas de las banderas de crecimiento a nivel global, pero esta tendrá éxito cuando se tienen indicadores de gestión que evalúan constantemente el desarrollo de la actividad o proceso que se esté midiendo buscando satisfacer las necesidades de los clientes y al mismo tiempo de nuestro entorno.

En el mismo orden de ideas la innovación se ha convertido hoy en día en factor relevante en el desarrollo de las organizaciones de clase mundial; este proceso permitirá a estás ser sustentable en el tiempo. Morales (2012) refiere que la gestión de la innovación son actividades dirigidas a resolver un problema extraordinario superable mediante pocas acciones o procedimientos, dando una gran importancia al evento de innovar como fuente de crecimiento para el sector industrial a nivel mundial.

Es por ello, que cuando se habla de innovación en las organizaciones en el mundo se generan cambios en todos los ámbitos, que se caracterizan por dar lugar a una continua e intensa colaboración entre grupos funcionales y profesionales que ponen de manifiesto su creatividad para obtener nuevos productos, procesos y servicios o mejora de los ya existentes. Por esta razón Getec (2013) manifiesta que gestionar la innovación es la organización del recurso humano con lo económico, con el fin de aumentar la creación de nuevos conocimientos e inventar nuevas ideas.

En tal sentido, realizar una gestión eficiente de la innovación influye de manera directa en que las organizaciones se mantengan a la vanguardia y con un alto nivel de competitividad, no solo a nivel tecnológico, sino también a través de la adquisición de nuevos conocimientos que le permitan al talento humano realizar de manera más eficiente y eficaz las actividades en pro de la rentabilidad de ellas.

En América latina busca adaptarse a estos nuevos esquemas con apoyo de organismos mundiales como la Organización para la cooperación y desarrollo (OCDE), Banco Mundial entre otros. Según Dutz (2019) indica "América Latina no ha estado lo suficientemente abierta al poder que representa la competencia del mercado en la generación de innovación" y, además, señala "Esto debería ser el semillero de una innovación constante" (p. 33).

No obstante, la industria petrolera nacional no se escapa de esta realidad por lo que gestionar la innovación se convierte en un reto importante para las organizaciones que habilitan los procesos medulares de la industria, que buscan ser competitivas y brindar calidad en la prestación de servicio; que contribuya al logro de los objetivos y metas planteados. La problemática de no innovar se presenta cuando los equipos de 
trabajo están cerrados en las viejas ideas, aptitudes y suposiciones que emplean para realizar sus actividades, sin tomar el tiempo de actualizar las metodologías de trabajo o se tiende a utilizar por defecto la solución que ve más como lo que ha funcionado en el pasado, en lugar de explorar nuevas ideas o forma distintas de realizar las cosas.

En consecuencia, las organizaciones de servicio de la industria petrolera buscan no perder su liderazgo y eficiencia, y por ende existe un gran compromiso para realizar las cosas tal y como las ha venido haciendo. El jugar a ganar o no jugar a perder, se convierte en su más grande desafío, sin permitir mirar más allá de lo que se puede hacer para mejorar los procesos. Transitar solo para apagar fuegos y sacar los mercados productos o servicios programado tan rápido como sea posible, pero realmente no se habla con el cliente, por lo tanto, no se escuchan las necesidades del mismo.

Actualmente, la gestión de la innovación en la industria petrolera en general, está a cargo de un equipo reducido de personas que se encargan de analizar las necesidades de innovar, este equipo de trabajo no necesariamente pertenece a las organizaciones que requieren hacer cambios novedosos en su forma de realizar las actividades y en muchos casos por desconocimiento de los procedimientos de trabajo puede $\circ$ no proponer soluciones que ayuden a mejorar los procesos. Según Ramírez y Peña (2011), la innovación requiere una combinación de habilidades y talento de todas las áreas de la organización.

A efecto de esta investigación y luego de un diagnostico que se realizó se ha podido establecer que en las gerencias de prestación de servicio de la industria petrolera cuales serán objeto de análisis hay situaciones donde ameritan ser examinadas; hay tecnologías donde requieren de actualización y se enmarcan en un nivel de obsolescencia, donde los procesos no están dando respuestas dentro del nuevo contexto organizacional, no se ha ido dando una actualización de programas y planes de trabajo situación está viene afectando los niveles de productividad y efectividad de la misma en tal sentido con esta investigación se busca dinamizar la implementación de aspectos tecnológicos que fundamente un cambio o una innovación.

Por lo tanto, existe la imperante necesidad de darle respuestas a los requerimientos de servicios en la industria petrolera; utilizando recursos que permitan darle un enfoque óptimo y en primer lugar el buen manejo de los procesos aplicando innovación, en la gerencia medular que habilitan los procesos de la industria petrolera tal como: Servicios Lacustre, Servicios Eléctricos, Automatización Informática y Telecomunicaciones, Mantenimiento y Construcción Lago.

No obstante, se presenta la interrogante de conocer si en las gerencias de prestación de servicios de la industria petrolera región occidente están a la vanguardia de la nueva tendencia en cuanto a gestión de la innovación. Basados en estos criterios se busca investigar sobre la gestión de la innovación en las gerencias de prestación de servicios de la industria petrolera. Es por ello que se pretende analizar la gestión de la innovación en las gerencias de prestación de servicios de la industria petrolera región Occidente.

\section{Gestión de la innovación}

La gestión de la innovación no es más que los pasos o estrategias que se siguen para llegar a obtener un resultado dramático: la 
innovación. Existen muchas formas de gestionar la innovación. Pero primero debe definirse porque se desea innovar, se establecen objetivos claros y cuál es el impacto que se espera. Todo esto ayuda a definir el tipo de innovación que se quiere lograr.

De acuerdo a Escorsa y Valls (2007) la innovación es el proceso en el cual a partir de una idea, invención o reconocimiento de una necesidad se desarrolla un producto, técnica o servicio útil hasta que sea comercialmente aceptado. En consonancia con lo expuesto por los autores el concepto de innovar no es más que el proceso de desarrollar algo nuevo, diferente, distinto o que no se conoce a partir del estudio metódico de una necesidad, ya sea personal, grupal u organizacional, para lograr una meta económica y de la mano con el éxito. Esto quiere decir, que la innovación genera ideas que pueden venderse en un mercado específico.

Asimismo, el nuevo paradigma de la gestión de la innovación del autor Maleria (2014) implica nuevas formas de concebir los nexos ahora más estrechos entre la competitiva de una empresa o corporación y sus opciones de innovación, desde un enfoque cíclico como altamente interactivo, desechando las aproximaciones lineales que subordinan y ponen en un segundo $o$ tercer término lo tecnológico. En este sentido, el mismo autor expone que dos elementos son fundamentales: llegar a asomarse a la frontera del conocimiento, tanto de lo posible (conocimiento científico) como de lo deseable (conocimiento mercadológico), en el objetivo seleccionado para de ahí, hacer surgir una estrategia ganadora.

De igual manera para Huber (2012) la gestión de la innovación es exitosa comienza cuando una solución inventiva resuelve un problema de mercado en el contexto de la estrategia de una empresa. La solución deberá ser inventiva ya que, de otra forma, los competidores la copiarán fácil y rápidamente y se perderá la ventaja competitiva. El problema de mercado deberá ser importante, para que el consumidor esté dispuesto a pagar el precio necesario para desarrollar los costos de desarrollo del producto $y$, finalmente, deberá está en el marco estratégico de la unidad de negocio porque, de lo contrario, carecerá de la tecnología necesaria, de los canales de mercadeo o de las habilidades de venta.

\section{Clases de innovaciones}

Indica Waissbluth (2015) existen diferentes clases de innovación; Una primera clasificación, es aquella que atiende al criterio del tipo de conocimiento en el que se sustenta la innovación, las clases de innovación se presentan según el objeto de la misma. También pueden referirse a la incorporación de nuevas funciones o características en servicios ya existentes, o a la introducción de servicios completamente nuevos, así como a mejoras sustanciales en la manera de provisión de los servicios. Se distinguen así, según se ha comentado anteriormente, innovaciones tecnológicas, innovaciones organizativas e innovaciones comerciales.

Según Sancho (2007) considera las clases de innovación como el resultado de un proceso lineal de flujo de conocimiento que iría desde la investigación científica básica siguiendo por el desarrollo experimental hasta la fabricación y comercialización del nuevo producto. Este modelo clásico concuerda con la teoría del impulso creado por la ciencia, según la cual, el desarrollo de ésta conduce a innovaciones que encuentran siempre un lugar 
en el mercado. Pero se podría adaptar igualmente a la teoría de la atracción ejercida por la demanda, ya que la demanda de un producto por los consumidores impulsa la actividad creativa e innovadora.

\section{Innovaciones tecnológicas de procesos} y servicios

Según Escorsa y Valls (2007) se basan en la utilización de nuevo conocimiento tecnológico o de nuevas tecnologías; o también pueden asentarse en nuevos usos o combinaciones de conocimiento o tecnologías ya existentes. Estas innovaciones se materializan en productos $\circ$ servicios tecnológicamente nuevos o mejorados con éxito en el mercado y también en procesos tecnológicamente novedosos que han sido incorporados a la producción o al suministro de forma eficiente. Para el Manual de Oslo (OCDE, 2005) indica que la innovación de producto aporta un bien o servicio nuevo, o significativamente mejorado, en cuanto a sus características técnicas o en cuanto a su uso u otras funcionalidades, la mejora se logra con conocimiento o tecnología, con mejoras en materiales, en componentes, o con informática integrada

\section{Innovaciones organizativas}

Por otra parte, Anzola (2015) indica que la innovación organizativa consiste en la aplicación de un nuevo método organizativo en las prácticas comerciales de la empresa, la organización del lugar de trabajo o las relaciones exteriores de la empresa. Por otra, según EUROSTAT y OCDE (2005), en el Manual de Oslo (2005), indica que la innovación en organización son cambios en las prácticas y procedimientos de la empresa, modificaciones en el lugar de trabajo, en las relaciones exteriores como aplicación de decisiones estratégicas con el propósito de mejorar los resultados mejorando la productividad o reduciendo los costes de transacción internos para los clientes y proveedores. La actualización en la gestión del conocimiento también entra en este tipo de innovación, al igual que la introducción de sistemas de gestión de las operaciones de producción, de suministro y de gestión de la calidad.

\section{Innovaciones comerciales}

A este respecto Waissbluth (2015) basadas en conocimientos de marketing, consisten en la creación y aplicación de un nuevo método de comercialización (marketing y venta) que conlleve cambios; por ser estas los generadores de conocimientos y estrategias hacia la mejora de la productividad y conseguir mejor calidad de vida, lo cual hace valorar a la ciudadanía en general.

Por otra parte, Escorsa y Valls (2007) manifiestan que el éxito comercial de un nuevo producto o servicio esencialmente depende de la superioridad del mismo sobre los restantes y del conocimiento del mercado y la eficacia del marketing desarrollado al efecto. Entre las innovaciones de dominio comercial destacan: nuevos medios de promoción de ventas, nuevas combinaciones estética-funcionalidad, nuevos sistemas de distribución y nuevas formas de comercialización de bienes y servicios. Un ejemplo de nuevas formas de comercialización es el sistema de franquicias o el comercio electrónico. Innovación organizativa: En este caso el cambio ocurre en la dirección y organización bajo la cual se desarrolla la actividad productiva y comercial de la empresa. 


\section{Etapas de un sistema de gestión de la innovación}

Según Vergara (2014) Se establecen cuatro etapas en este proceso, que hacen de este sistema, un proceso circular virtuoso, pues en la medida que el ciclo se repita recurrente y recursivamente, se logrará en cada ciclo, obtener una mejora. Las cuatros etapas del sistema de gestión son: Ideación, Planeación, Implementación y Control.

Para Aponte (2016) la gestión de la innovación tecnológica engloba varias etapas y cada etapa contiene varios procesos que permiten gestionar el proceso global desde el inicio de la idea hasta su transformación en la innovación per se, cuando ésta se encuentra disponible en el mercado. Estos procesos están presentes en todo el ciclo de desarrollo de la innovación; donde estas etapas se pueden resumir en la concepción, implementación y mercadeo de la innovación.

\section{Ideación}

Según Vergara (2014) el objetivo de esta etapa es trabajar en la idea que guiará los primeros pasos del proceso de creación que se logra con el sistema de gestión propuesto.

\section{Planeación}

En este aspecto Vergara (2014) indica que dentro del proceso de la planificación constituye una etapa fundamental y el punto de partida de la acción directiva, ya que supone el establecimiento de sub-objetivos y los cursos de acción para alcanzarlos. En esta etapa, se definen las estrategias que se utilizarán, la estructura organizacional que se requiere, el personal que se asigna, el tipo de tecnología que se necesita, el tipo de recursos que se utilizan y la clase de controles que se aplican en todo el proceso.
Francés (2016) la planificación tecnológica estratégica es un proceso en el cual se definen de manera sistemática los lineamientos estratégicos de la organización, las líneas detalladas para la acción, los recursos asignados y plasmados en documentos llamados planes. Es un proceso que pretende alcanzar un futuro deseable a partir del análisis de la realidad existente interna y externa y de las capacidades de la entidad, orientado a la acción y en la que participa toda la organización.

\section{Implementación}

Vergara (2014) manifiesta en un contexto empresarial, esto se refiere a la dirección que toman las decisiones y las acciones para alcanzar los objetivos trazados. Es importante destacar que las decisiones y acciones que se toman para llevar adelante un propósito, se sustentan en los mecanismos 0 instrumentos administrativos (estrategias, tácticas, procedimientos, presupuestos, entre otros.), que están sistémicamente relacionados y que se obtienen del proceso de planificación.

\section{Control}

Según Sancho (2007) es necesario disponer de indicadores que reflejen las actividades de innovación, que indiquen los factores que influyen o dificultan su capacidad innovativa. Como se ha dicho, durante mucho tiempo se han considerado los gastos en $\mathrm{I}+\mathrm{D}+\mathrm{i}$ como indicadores de los procesos innovadores y las solicitudes y concesiones de patentes como medida directa de los resultados, pero se ha demostrado, de esta manera se obtiene control de los procesos de innovación importante para la toma de decisiones. 
Finalmente, Zabala (2016) estipula que el control busca calidad en la organización, permite verificar que los productos o servicios ofrecidos por las empresas reúnan las condiciones necesarias para su provechosa, sana y confiable utilización, de acuerdo a lo ofrecido. Por lo tanto, es un análisis que se hace a fin de asegurar que un producto 0 servicio sea ejecutado con la mayor excelencia posible en el contexto empresarial, por lo que debe estar acorde a un sistema de gestión de la calidad.

\section{Factores determinantes en la gestión de la innovación}

Para Drucker (2002) el primer punto es que la innovación = invención + explotación. El proceso de invención cubre todos los esfuerzos dirigidos a crear nuevas ideas y ponerlas en funcionamiento. El proceso de explotación supone las fases de desarrollo comercial, de aplicación y de transferencia, lo cual incluye la orientación de las ideas o de las invenciones hacia objetivos específicos, la evaluación de dichos objetivos, la transferencia de los resultados de investigación $y / o$ desarrollo, y la futura utilización y difusión de dichos resultados.

Por otro lado, para Sancho (2007) indica que la evolución de las necesidades de los consumidores, el desarrollo de nuevas tecnologías, y la liberalización del comercio internacional, entre otros, son factores determinantes en la gestión de la innovación que han originado en las últimas décadas profundos cambios en la estructura, estrategia, actividades y funcionamiento de las industrias. Por otra parte, la globalización ha aumentado notablemente el acceso a la información y ha abierto nuevos mercados, lo que ha llevado a una mayor competitividad internacional.

\section{Personal}

Según, OECD (2005) la necesidad de empoderar a las personas para innovar, requiere una educación adecuada y de alta calidad, además del desarrollo de una amplia gama de habilidades como complemento de la educación formal. Los planes de estudio y la enseñanza deberán adaptarse con objeto de que los estudiantes estén preparados para aprender y aplicar nuevas habilidades a lo largo de su vida. El capital humano es la parte fundamental de la innovación. El empoderamiento de las personas para innovar depende de una educación amplia y adecuada, además de desarrollar un amplio rango de habilidades que complementan la educación formal. Los planes de estudio y las formas de enseñanza deberán adaptarse a fin de brindar a los estudiantes capacidades para aprender y aplicar nuevas habilidades a lo largo de su vida.

Para Hidalgo (2014) además de las cuestiones gerenciales relativas a los papeles y personas necesarios para la creación de una plantilla eficiente, existen otras que afectan a la productividad del personal, tanto desde el punto de vista individual como del grupo de trabajo. Las diferentes fases por las que atraviesa la carrera de un ingeniero o científico y la composición de su grupo de trabajo inmediato ejercen gran influencia sobre su productividad técnica.

\section{Estructura}

Para Hidalgo (2014) el diseño de estructuras organizativas que incrementen la capacidad de innovación técnica requiere centrarse en las aportaciones externas que recibe es organización y los resultados finales que produce. Una organización de $I+D+1$ eficaz necesita unas aportaciones apropiadas 
de información técnica y de mercado y precisa que sus resultados estén integrados dentro de los objetivos generales y que sean transferidos hacia el usuario final.

Dependiendo del tipo de empresa de que se trate $y$ del sector industrial al que pertenezca, entre uno y dos tercios de dichos clientes pertenecen al departamento de producción de la empresa de que realiza el desarrollo. Producción decidirá comprar o no la mejora desarrollada en materiales, componentes, equipamiento $\bigcirc$ procesos productivos para su propio uso interno. $Y$ este posible cliente necesita al menos el mismo grado de participación en el proceso de diseño y desarrollo que una persona o empresas externas.

\section{Estrategia}

Según Hidalgo (2014) la gestión de la tecnología incluye aspectos de planificación y de implantación estratégica que pueden situarse en dos niveles: el general en toda la empresa, agencia de la administración, división o línea de productos; y el particular más centrado en el proceso/departamento /laboratorio de la organización, dedicado al desarrollo y a la adquisición de tecnología.

El mismo autor, señala que se ha demostrado que la evolución de una tecnología tiende a seguir un proceso que consta de tres fases, y cada fase supone diferentes implicaciones estratégicas. La primera fase tiende a mostrar frecuentes e importantes innovaciones de producto, que en general surgen en pequeños grupos de trabajo emprendedores y que a menudo estén muy ligados a las necesidades de usuarios tecnológicamente avanzados.

La fase intermedia suele mostrar grandes innovaciones de proceso, una continua variación del producto y un creciente número de competidores, tanto grandes como pequeños. La última fase presenta menos frecuentes innovaciones de producto $y$ proceso, realizadas principalmente por grandes empresas cuya motivación principal es satisfacer objetivos operativos de reducción de costes y mejora de la calidad.

\section{MÉTODO}

En consonancia con el objetivo de investigación, se ubicó el estudio dentro del tipo descriptivo, con un diseño no experimental, de campo y transeccional. Para los fines se consideró como población a las gerencias de prestación de servicios de la industria petrolera región occidente, la cual quedó representada por cuatro (4) gerencias activas al momento de la recolección de datos, siendo estas: Servicios Lacustre, Servicios Eléctricos, Mantenimiento Lago y Automatización Informática y Telecomunicaciones, cuyos sujetos informantes fueron gerentes, líderes $y$ supervisores del área, como se muestra en la Tabla 1. 
Tabla 1. Población de estudio

\begin{tabular}{|c|c|c|c|}
\hline GERENCIA & GERENTES & LÍDERES & SUPERVISORES \\
\hline Servicios Lacustres & 1 & 2 & 3 \\
\hline Servicios Eléctricos & 1 & 2 & 3 \\
\hline Mantenimiento Lago & 1 & 2 & 3 \\
\hline Automatización, Información y Telecomunicaciones & 1 & 2 & 3 \\
\hline SUB-TOTAL & 4 & 8 & 12 \\
\hline TOTAL & & 24 & \\
\hline
\end{tabular}

Fuente: Elaboración propia

Otro aspecto clave, a desarrollar está relacionado con la definición de la técnica e instrumentos de recolección de información, fue la encuesta como técnica, y el instrumento de recolección de datos, en este caso el cuestionario diseñado con base a los objetivos planteados, conformado por 31 ítems, de acuerdo al nivel de frecuencia con el que se cumplen tales afirmaciones y las Opciones de: siempre, casi siempre, algunas veces, casi nunca y nunca.

El instrumento diseñado se sometió a un proceso de validación de contenido, a través de la técnica del Juicio de Expertos, a cinco (5) profesionales en el área de gestión de la innovación (3) y metodológica (2). Se realizó el cálculo de confiabilidad al instrumento diseñado para medir la variable gestión de la innovación obteniendo un coeficiente Alpha de Cronbach de 0,79, donde los resultados demostraron que el instrumento, según los rangos y magnitudes mencionados, era aceptable e idóneo para ser aplicado, teniendo alta confiabilidad.

Para procesar los resultados se recurrió al método de la estadística descriptiva, el análisis se realizó con base a las medias aritméticas o promedio. Ahora bien, para la interpretación de los resultados, el investigador diseñó dos (2) baremos, correspondientes al análisis de la media aritmética de las dimensiones e indicadores, y otro para la variable, en los cuales se muestra el rango $o$ intervalo para cada alternativa de respuesta, categoría asignada, y descripción de la misma, tal como se muestra en las Tablas 2 y 3 . 
Tabla 2. Baremo para la interpretación de las dimensiones e indicadores

\begin{tabular}{|c|c|c|}
\hline INTERVALO & CATEGORÍA & DESCRIPCIÓN \\
\hline $4.21-5$ & Muy alta aplicación & Si la aplicación es muy alta o alta se considera una \\
\hline $3.41-4.20$ & Alta aplicación & fortaleza para la gestión de la innovación \\
\hline $2.61-3.40$ & Moderada aplicación & $\begin{array}{l}\text { Si la aplicación es moderada se considera una leve } \\
\text { fortaleza, la gestión de la innovación requiere mejoras }\end{array}$ \\
\hline $1.81-2.60$ & Baja aplicación & $\begin{array}{l}\text { Si la aplicación es baja o muy baja se considera una } \\
\text { debilidad para la gestión de la innovación, por tanto }\end{array}$ \\
\hline $1-1.80$ & Muy baja aplicación & requiere mejoras. \\
\hline
\end{tabular}

Fuente: Elaboración propia

Tabla 3. Baremo para la interpretación de la variable

\begin{tabular}{ccc}
\hline INTERVALO & CATEGORÍA & DESCRIPCIÓN \\
\hline $4.21-5$ & Muy alta aplicación & Indica buena gestión de la innovación \\
$3.41-4.20$ & Alta aplicación & \\
$2.61-3.40$ & Moderada aplicación & Indica regular gestión de la innovación \\
$1.81-2.60$ & Baja aplicación & \\
$1-1.80$ & Muy baja aplicación & Indica mala gestión de la innovación \\
\hline
\end{tabular}

Fuente: Elaboración propia

RESULTADOS

Así entonces, en la Tabla 4, se presenta el promedio reportado para la dimensión clases de innovaciones donde para el indicador las innovaciones tecnológicas de procesos y servicios según la opinión de los encuestados se alcanzó un promedio de 2,56 de manera general que indica baja aplicación y es considerada como una debilidad para la gestión que se analiza, debido a que casi nunca se incorpora nuevos conocimientos en sus procesos, que permitan mejorar el servicio prestado por su organización. Se evidencia que las gerencias de prestación de servicios en la industria petrolera investigadas deben cumplir con las exigencias actuales a nivel mundial donde prevalece la gestión del conocimiento para proporcionar mejoras continuas y de esta manera profundizar en la organización la innovación como eje medular.

Los hallazgos encontrados difieren de lo establecido por Manual de Oslo (OCDE, 2005), indicando que la innovación de producto aporta elementos importantes a los bienes o servicio nuevo, o mejorado, en cuanto a sus características, uso u funciones. 
Tabla 4. Clases de las innovaciones

\begin{tabular}{lcll}
\hline \multicolumn{1}{c}{ INDICADOR } & $\mathrm{X}$ & CATEGORÍA & \multicolumn{1}{c}{ INTERPRETACIÓN } \\
\hline $\begin{array}{l}\text { Las innovaciones tecnológicas } \\
\text { de procesos y servicios }\end{array}$ & 2,56 & Baja aplicación & $\begin{array}{l}\text { Se considera una debilidad para la } \\
\text { Organizativas }\end{array}$ \\
Comerciales & 2,54 & Baja aplicación de la innovación, por tanto \\
Total dimensión: & 2,55 & Baja aplicación & $\begin{array}{l}\text { Baja aplicación } \\
\text { requeras. }\end{array}$ \\
\hline
\end{tabular}

Fuente: Elaboración propia

En cuanto al indicador innovaciones organizativas, se evidencia que existe una baja aplicación con un promedio general de 2,54, para la gestión analizada. A este respecto se considera que casi nunca se están generando estructuras de trabajo adecuadas, así como las relaciones externas e internas tiene una conducción acertada y las prácticas de negocio no tienen una visión holística que genere innovación. A este respecto, Anzola (2015) indica que la innovación organizativa consiste en la aplicación de un nuevo método organizativo en las prácticas comerciales de la empresa, la organización del lugar de trabajo o las relaciones exteriores de la empresa. En este sentido los resultados obtenidos están en desacuerdo con lo que plantea el autor, la organización debe estar orientada a dirigir prácticas y generar estrategias, procedimientos innovadores para estar en vanguardia y ser competitivos esto va contribuir a la mejora de prestación de servicios y de alguna generar un impacto positivo en la productividad.

En lo que respecta al indicador innovaciones comerciales, los resultados arrojaron baja aplicación siendo una debilidad en ese proceso de 2,54, debido a que casi nunca la organización hace públicos los beneficios de los servicios que presta e igualmente se promociona el catálogo de servicio en las gerencias analizadas. Según lo indicado por Escorsa y Valls (2007) manifiestan que el éxito comercial de un nuevo producto $\mathrm{o}$ servicio esencialmente depende de la superioridad del mismo sobre los restantes y del conocimiento del mercado y la eficacia del marketing desarrollado al efecto. En este sentido con los resultados obtenidos se denota el desconocimiento de la clase de innovación comercial analizado, generando esto una debilidad en las gerencias analizadas para generar valores innovadores en los productos de servicios prestados dejando de percibir cualquier beneficio pudiendo quedar lejos la de competitividad.

En relación a los resultados correspondientes a la dimensión clases de las innovaciones, según la opinión de los encuestados se ve reflejada una media de 2,55 , indicando debilidades para la gestión en cuanto a las clases: Las innovaciones tecnológicas de procesos y servicios, organizativas y comerciales. Implicando, a su vez, baja aplicación en esa dimensión lo cual denota que las gerencias de prestación de 
servicio de la industria petrolera casi nunca están a la vanguardia en ningunos de esos términos siendo este preocupante ya que no se están generando ni empleando innovaciones de ninguna índole.

Según Sancho (2007), considera las clases de innovación como el resultado de un proceso lineal de flujo de conocimiento que iría desde la investigación científica básica siguiendo por el desarrollo experimental hasta la fabricación y comercialización del nuevo producto. En este sentido, las clases de las innovaciones en las gerencias de prestación de servicios en la industria petrolera requieren de una revisión profunda con el fin de mejorar todos los procesos productivos; la prestación de servicios puede incluir mejoras significativas en la manera en que estos servicios se prestan (en términos de eficiencia y rapidez).
En cuanto a la dimensión etapas de un sistema de gestión de la innovación, en la tabla 5, se muestra los resultados obtenidos del trabajo de campo, donde se observa para el indicador ideación una media de 3,39, indicando una moderada aplicación en las etapas de un sistema de gestión de las gerencias de prestación de servicios de la industria petrolera, implicando una leve fortaleza para la gestión analizada. Esto debido a que algunas veces se establecen planes estratégicos donde surgen ideas entre el equipo para mejorar la prestación de servicio e igualmente el departamento de gestión de propuestas tiene una metodología para lograr definir una idea mejorando el servicio.

Tabla 5. Etapas de un sistema de gestión

\begin{tabular}{|c|c|c|c|}
\hline INDICADOR & $\mathrm{x}$ & CATEGORÍA & INTERPRETACIÓN \\
\hline Ideación & 3,39 & Moderada aplicación & \\
\hline Planeación & 3,35 & Moderada aplicación & Se considera una leve fortaleza, la gestión \\
\hline Implementación & 3,33 & Moderada aplicación & \\
\hline Control & 3,33 & Moderada aplicación & \\
\hline Total dimensión: & 3,35 & Moderada aplicación & $\begin{array}{l}\text { Se considera una leve fortaleza, la gestión } \\
\text { de la innovación requiere mejoras }\end{array}$ \\
\hline
\end{tabular}

Fuente: Elaboración propia

Según Vergara (2014), el objetivo de esta etapa es trabajar en la idea que guiará los primeros pasos del proceso de creación que se logra con el sistema de gestión propuesto. En consecuencia con lo referido por el autor; se refleja que a veces se está abordando en la organización mecanismos de creatividad, se tienen que buscar criterios firmes dentro de la organización para impulsar la generación de ideas dando paso a la invención y luego obtener ese producto innovador para dar una prestación de servicio óptima. 
Ahora bien, para el indicador Planeación, se observa una moderada aplicación del mismo en la gestión de la innovación que se analiza, cuya media se posicionó en 3,35; indicando que la gestión posee leve fortalezas en cuanto a lo medido en este indicador. Al observar el comportamiento de los ítems aplicados, se denota moderada aplicación de estrategias funcionales, lineamientos corporativos entre otros.

A este respecto señala Francés (2016), la planificación tecnológica estratégica define de manera sistemática los lineamientos estratégicos de la organización, las líneas detalladas para la acción, los recursos asignados y plasmados en documentos llamados planes.

Al detallar los resultados plasmados para el indicador implementación, se observa una moderada aplicación con una media general de 3,33, indicando que con moderada frecuencia se realizan actividades que generan valor agregado innovador dentro de las organizaciones, en el estudio es visible que se emplean métodos que permiten gestionar nuevos conocimientos y hacen chequeos rutinarios a los manuales y procedimientos en cada gerencia, delineando estos aspectos como leve fortalezas de la gestión.

No obstante, indica Vergara (2014) manifiesta que las decisiones y acciones que se toman para llevar adelante un propósito, se sustentan en los mecanismos 0 instrumentos administrativos (estrategias, tácticas, procedimientos, presupuestos, entre otros.), que están sistémicamente relacionados y que se obtienen del proceso de planificación. Considerándose el autor mencionado, las organizaciones estudiadas algunas veces están siendo efectivas en la aplicación de estrategias innovadoras para implementar tácticas vanguardistas que aceleren procedimientos $y$ mejoren los presupuestos en cada una de ellas.

De acuerdo a los valores para el indicador control, se observa moderada aplicación del mismo en la gestión de la innovación que se analiza, cuya media se posicionó en 3,33; indicando que la gestión posee leves fortalezas en cuanto a lo medido en este indicador. Al observar el comportamiento, se denota moderada aplicación en cuanto a si utilizan métodos de control que permitan determinar las desviaciones, en los procesos de las gerencias de prestación de servicios en la industria petrolera región occidente.

Al comparar estas evidencias con lo planteado por Zabala (2016), estipula que el control busca calidad en la organización, permite verificar que los productos o servicios ofrecidos por las empresas reúnan las condiciones necesarias para su provechosa, sana y confiable utilización, de acuerdo a lo ofrecido. De igual manera, valida el investigador quien opina que, el control es considerado un elemento clave en la gestión de la innovación, el cual permite detectar las posibles desviaciones que se pueden presentar en la prestación de servicios dando parte a la organización para lograr solucionarlas en consonancia con los requerimientos establecidos.

Los resultados correspondientes a la dimensión etapas de un sistema de gestión, en la cual se ve reflejada una media de 3,35, indicando leve fortaleza en la gestión de la innovación en cuanto a las etapas: ideación, planeación, implementación y control. Según Aponte (2016), indica que la gestión de la innovación tecnológica engloba varias etapas y cada etapa contiene varios procesos que permiten gestionar el proceso global desde el inicio de la idea hasta su transformación en la innovación per se, cuando ésta se encuentra disponible en el mercado. 
En cuanto a la dimensión factores determinantes en la gestión de la innovación, sus resultados se reflejan en la Tabla 6 , al detallar los hallazgos reflejados para el indicador personal se observa que existe moderada aplicación, con un promedio general de 3,35, indicando que algunas veces se realizan inversiones en programas I+D, asimismo, el desarrollo de carrera es evaluado constantemente y hacen reuniones dentro de la organización para visualizar estrategia innovadoras y dar respuesta a los problemas que se presentan en un momento especifico, delineando estos aspectos como leve fortalezas de la gestión y que requiere mejoras al respecto.

Tabla 6. Factores determinantes en la gestión de la innovación

\begin{tabular}{cccl}
\hline INDICADOR & $\mathrm{X}$ & CATEGORÍA & \multicolumn{1}{c}{ INTERPRETACIÓN } \\
\hline Personal & 3,35 & Moderada aplicación & $\begin{array}{l}\text { Se considera una leve fortaleza para la gestión } \\
\text { de la innovación }\end{array}$ \\
Estructura & 1,81 & Baja aplicación & $\begin{array}{l}\text { Se considera una debilidad para la gestión de la } \\
\text { innovación, por tanto requiere mejoras. }\end{array}$ \\
Estrategia & 1,93 & Baja aplicación & $\begin{array}{l}\text { Se considera una debilidad para la gestión de la } \\
\text { innovación, por tanto requiere mejoras. }\end{array}$ \\
Total dimensión: & 2,36 & Baja aplicación & $\begin{array}{l}\text { Se considera una debilidad para la gestión de la } \\
\text { innovación, por tanto requiere mejoras. }\end{array}$ \\
\hline
\end{tabular}

Fuente: Elaboración propia

Así entonces, es pertinente lo planteado según, OECD (2005), la necesidad de empoderar a las personas para innovar, requiere una educación adecuada y de alta calidad, además del desarrollo de una amplia gama de habilidades como complemento de la educación formal.

Seguidamente, se muestran los resultados obtenidos para el indicador estructura, indicando baja aplicación como parte de los factores determinantes en la gestión de la innovación que aplican las organizaciones, con un promedio de 1,81 indicando debilidad de la gestión, ya que casi nunca poseen herramientas que incrementen la capacidad técnica y promueven la innovación creativa.

Estos resultados permiten afirmar que las organizaciones estudiadas casi nunca están acorde con lo planteado por Hidalgo (2014), cuando expresa que las estructuras organizativas permiten incrementar la capacidad de innovación técnica por las aportaciones externas que recibe y los resultados finales que produce.

De acuerdo a los valores relacionados con el indicador estrategia, se tiene una media de 1,93 indicando baja aplicación del indicador. Visto así, en las gerencias analizadas el tipo de factor determinante en la gestión la innovación casi nunca se ejecuta las estrategias para la mejora en cuanto a la prestación del servicio, de tal manera que se constituye en una debilidad por tanto requiere mejoras. Las evidencias anteriores contradicen el postulado de Hidalgo (2014), quien ha demostrado que la evolución de una tecnología tiende a seguir un proceso que consta de tres fases, y cada fase supone diferentes implicaciones estratégicas. La primera fase tiende a mostrar frecuentes e importantes innovaciones de 
producto, la otra suele mostrar grandes innovaciones de proceso, y la última presenta menos frecuentes innovaciones de producto $y$ proceso.

De manera general para la dimensión factores determinantes en la gestión de la innovación, se observa un promedio general de 2,36 ubicándose en una baja aplicación de los factores en la gestión analizada, dado a que solo en el indicador personal resulto la categoría de moderada aplicación siendo de $(3,35)$ el resto de los indicadores como estructura, estrategia arrojaron $(1,81)$ y $(1,93)$ respectivamente, entrando todos en la interpretación de una debilidad para la gestión de la innovación.

Las evidencias anteriores, de baja aplicación, van en contra del postulado teórico de Sancho (2007), quien indica que la evolución de las necesidades de los consumidores, el desarrollo de nuevas tecnologías, y la liberalización del comercio internacional, entre otros, son factores determinantes en la gestión de la innovación que han originado en las últimas décadas profundos cambios en la estructura, estrategia, actividades y funcionamiento de las industrias.
También validan al investigador cuando afirma que los factores determinantes en la gestión de la innovación tienen que estar en la búsqueda proactiva de elementos técnicos o de mercado aprovechables para que los esfuerzos desarrollados en la organización sean notables y en los resultados es todo lo contrario donde se requiere de manera imperante una corrección.

En la Tabla 7 se muestra los resultados para la variable gestión de la innovación, alcanzando una media general de 2,74, ubicándose en la categoría de moderada aplicación, indicando regular gestión de la innovación. En este sentido, en la gestión de la innovación de las gerencias de prestación de servicios de la industria petrolera región occidente, se adjudica baja aplicación a las clases de innovación con $(2,54)$, a las etapas de un sistema de gestión $(3,33)$ la clasifica como moderada, y a los factores determinantes en la gestión de la innovación $(2,36)$ resultando baja aplicación, convirtiendo estos aspectos en una leve fortaleza para de la gestión.

Tabla 7. Gestión de la innovación

\begin{tabular}{|c|c|c|c|}
\hline DIMENSIÓN & $X$ & CATEGORÍA & INTERPRETACIÓN \\
\hline Clases de innovación & 2,54 & Baja Aplicación & $\begin{array}{l}\text { Se considera una debilidad para la gestión de la } \\
\text { innovación, por tanto requiere mejoras. }\end{array}$ \\
\hline $\begin{array}{l}\text { Etapas de un sistema de } \\
\text { gestión }\end{array}$ & 3,33 & $\begin{array}{l}\text { Moderada } \\
\text { Aplicación }\end{array}$ & $\begin{array}{l}\text { Se considera una leve fortaleza, la gestión de la } \\
\text { innovación requiere mejoras }\end{array}$ \\
\hline $\begin{array}{l}\text { Factores determinantes en la } \\
\text { gestión de la innovación }\end{array}$ & 2,36 & $\begin{array}{l}\text { Baja } \\
\text { Aplicación }\end{array}$ & $\begin{array}{l}\text { Se considera una debilidad para la gestión de la } \\
\text { innovación, por tanto requiere mejoras. }\end{array}$ \\
\hline Total variable & 2,74 & $\begin{array}{l}\text { Moderada } \\
\text { aplicación }\end{array}$ & Indica regular gestión de la innovación \\
\hline
\end{tabular}

Fuente: Elaboración propia 
Los resultados dados moderadamente validan lo dicho por Escorsa y Vals (2007), quienes la definen como los pasos 0 estrategias que se siguen para llegar a obtener un resultado dramático: La innovación. Los mismos autores, refiere que existen muchas formas de gestionar la innovación, pero primero debe definirse por que se desea innovar, establecer objetivos claros y cuál es el impacto que se espera.

De igual manera, valida al investigador cuando considera que, lo expuesto por los autores el concepto de innovar no es más que el proceso de desarrollar algo nuevo, diferente, distinto o que no se conoce a partir del estudio metódico de una necesidad, ya sea personal, grupal u organizacional, para lograr una meta económica y de la mano con el éxito. Esto quiere decir, que la innovación genera ideas que pueden venderse en un mercado específico.

\section{CONCLUSIONES}

Los resultados del presente estudio permiten dar respuesta al propósito de analizar la gestión de la innovación en las gerencias de prestación de servicios de la industria petrolera región Occidente, se presenta una regular gestión de la innovación en las gerencias analizadas, argumentos tanto resaltantes como significativos los cuales permitieron llegar a las siguientes conclusiones presentadas a continuación:

En relación a las clases de innovación desarrolladas en las gerencias de prestación de servicios en la industria petrolera región Occidente, reflejados una media de 2,54, indicando debilidades para la gestión en cuanto a las clases de las innovaciones tecnológicas de procesos y servicios, organizativas y comerciales. Implicando, a su vez, lo cual denota que las gerencias de prestación de servicio de la industria petrolera casi nunca están a la vanguardia en ningunos de esos términos siendo este preocupante, por cuanto no se están generando ni empleando innovaciones de ninguna índole.

Referente a las etapas de un sistema de gestión de la innovación en las gerencias de prestación de servicios en la industria petrolera región occidente, arrojando una media de 3,33 , indicando leve fortaleza en la gestión de la innovación en cuanto a las etapas: ideación, planeación, implementación y control, lo cual sin duda alguna es moderada aplicación para las organizaciones involucradas.

Concerniente a los factores determinantes en la gestión de la innovación en las gerencias de prestación de servicios en la industria petrolera región Occidente, reflejando un promedio de 2,36 encontrándose una baja aplicación de los factores para la gestión de la innovación, indicando ello la debilidad de los elementos en las áreas analizadas.

\section{REFERENCIAS}

Anzola (2015). Evaluación de Proyectos. Primera Edición. Editorial McGraw-Hill. México

Aponte (2016). Procedimientos Administrativos y Operativos. Ediciones Panapo. Caracas - Venezuela

Drucker (2002). Gestión de Empresas: Planificación, Ejecución y Control. Editorial Limusa Willey

Dutz, H (2019). Gestión de Operaciones. Editorial Mc. Graw Hill

Escorsa y Valls (2007). Procedimientos Operativos a Nivel Empresarial. Editorial McQuenn. México. D-F

Francés (2016). Optimización de Procesos Productivos. Editorial McGraw Hill. DF México 
Getec (2013). Mejores Prácticas Logísticas en Latinoamérica. Editorial: International Thomson

Hidalgo (2014). Guía de Técnica Moderadas de Administración de Empresas. Editorial Pearson Prentico Hall. México

Huber (2012). Desarrollo de Sistemas Productivos. (Primera Edición). Ediciones UPC. Barcelona, España

Maleria (2014). Logística: Optimización de Tiempo y Productividad. Editorial Pearson Educación. Madrid España

Morales, N (2012). Herramientas Para los Procesos de Calidad. Editorial Roma. Venezuela - Caracas

Manual de Oslo. (2005). Guía para la recogida e interpretación de datos sobre innovación. Tercera edición OECD/European Communities, ISBN 84-611-2781-1. (C) TRAGSA 2006

Ramírez y Peña (2011). Planificación de Requerimientos Productivos. México: Editorial McGraw-Hill

Sancho (2007). Evaluación de Proyectos Productivos. Editorial McGraw-Hill. México

Vergara (2014). Introducción al Estudio del Trabajo. Ediciones 21. Madrid - España Waissbluth (2015). Procesos Organizativos. (Segunda Edición). Bogotá Colombia: Editorial Mc Graw Hill

Zabala (2016). Administración Empresarial, Una Perspectiva Global. DF - México. McGraw Hill 